\title{
A Study on Fire Detection System using Statistic Color Model
}

\author{
Megha Jess Mathew \\ Assistant Professor, Dept: Of ECE,MVJ College of Engineering, Near ITPB, Channasandra, Bangalore-67 \\ 09060101159
}

\begin{abstract}
Normally fire detection system uses the heuristic fixed threshold values in their specific methods. However, input images may be changed, in general, so the heuristic fixed threshold values used in the fire detection systems might be modified on a case by case basis. In this paper, an automatic fire detection system without the heuristic fixed threshold values was studied. We presented an automatic method using the statistical color model and the binary background mask. We did the experiment using 600 frames from 6 typical different fire video clips. As the experimental results the proposed method showed a good performance of about average $85 \%$ detection rate without false positive, compared with the other methods with the heuristic fixed threshold values.
\end{abstract}

Keywords: Emperical value,Fire detection, RGB,threshold,sensors

\section{Introduction}

Fire is necessary and profitable to humankind life, but it also causes the damage of life and property in certain case. According to Korean National Emergency Management Agency, in January 2006, when we consider the fire occurrence number of 3,357 cases, the loss of lives of 251 people, the property damage of 17,600 million wons, after one year later, in January 2007 , it showed monthly about 4,877 fire cases, the loss of lives of 252 people, the property damage of 19,500 million wons. Therefore, the fire number showed about $45 \%$ increase and a loss of lives is almost no change, but about $11 \%$ of property damage increased [1]. In general, the early presence of fire can be detected by smoke, gas or flame. Most of the fire detection detects the fire based on sensors. However, a sensor can be not reliable and fast because there can be time delay with the sensor [2]. If the threshold value is low, the sensor can have high sensitivity but it can produce false alarm. And it also requires that a certain threshold value must be assign to each sensor. On the other hand, the image processing-based fire detection has various advantages [2]. Light travels very high speed, so the image processing-based fire detection can be quick to catch the spot of fire on time without delay. The image processing-based fire detection system can also monitor wider space than the conventional sensor-based fire detection system. There are many researches on the image processing-based fire detection systems. To detect fire, only using color information may produce false alarm, so color and temporal variation information should be used to get a good performance of a fire detection system. Some researches used RGB input and the simple and effective procedure for real-time application [3-5]. Others adopted panoramic camera, wavelet transform, neural networks, etc. for relatively complicated fire detection systems [6-8]. And some were very specific purposed systems like tunnel fire, ship compartmentfire, forest fire, etc [9-11]. So it is not easy to compare the fire detection systems directly. In 2004, the fire detection method proposed by Chen et al. adopted the RGB color based chromatic model and used disorder measurement. They used the intensity and saturation of red component and the segmentation by image differencing [3]. The method is simple but they have the heuristic fixed threshold values of chromatic information. In 2007, Lee and Han also used the RGB color input video for real-time fire detection in the tunnel environment with many predetermined threshold values [4]. In 2007, Ceilk et al. studied the fire detection method using the statistical color model and foreground object information. They introduced the statistic colormodel for generic fire model. However, when they calculated the color channel ratio to eliminate the luminance component for the color based fire classification [5], they still used the heuristic fixed threshold values. Most of above mentioned researches used the heuristic fixed thresholds in their fire detection systems. And also the experimental environment was so limited like a tunnel. In this paper, they tried to exclude the heuristic fixed threshold values used in the fire detection method byChen et al. And they want to solve a problem that reliability may drop by the use of the heuristic fixed values in the limited environment. That is, they replaced the heuristic fixed threshold values with the statistic threshold values so that the automatic calculation without the empirical fixed threshold values is possible.

\section{Related Research of Fire Detection System}

\subsection{Problem of color based detection with heuristic threshold}

In general, flame displays various color ranges from red color to yellow according to the burning materials. Therefore, the fire detection system based on the input color, usually gives the heuristic threshold 
values to find fire to the RGB input color [3-5]. Some used the red color with a heuristic threshold value [3]. In this case, if the color of fire is changed, the fire detection is not easy. One of typical example of these approaches is that of Chen's group. They detected fire by using the heuristic fixed threshold values. For a generic fire color model, they should be replaced with automatic threshold values using the statistics color model.

\subsection{Method based on color model}

In Chen's method, the heuristic fixed threshold value of red color can be replaced with the statistic value using the statistic color model. In 2007, T. Celik et al. studied the fire detection using the statistical color model in video sequences [5]. They replaced the red color threshold value with the statistical model value, the average value, but their method still used six heuristic threshold values to remove the luminancecomponent of the RGB color space by the color channel ratio. In this case, their heuristic threshold values for eliminating the luminance [5] were good for the experiment of the specific fire detection in the woods. However, it still had the heuristic fixed threshold values in the fire detection system. Here,they tried to remove the usage of the heuristic threshold values in our fire detection system.

\subsection{Method with statistical color model and BBM}

To avoid the heuristic fixed threshold values in the fire detection system, they replaced the heuristic values in Chen's method with the statistic values and the binarybackground mask (BBM). That is, first, they got the difference of the input frame and the normal frame, and get the binary background mask from the difference image frame. They calculated the average of red component and the average of saturation in the fire candidate region by using BBM. This approach can remove the fire-like color regions of background from the image frames of video sequences.

\section{Fire Detection System with Binary Background Mask}

In Figure 1, the proposed fire detection system using the statistical threshold values and BBM is described.

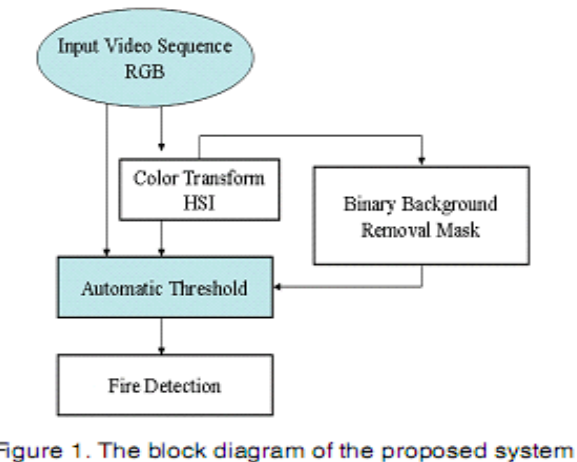

At first, the input RGB video sequences are inputted to the fire detection system. RGB video frames are captured for the fire detection. HSI transform is done for getting the binary background mask through the frame difference. Then the automatic threshold values can be acquired from the statistical color model and BBM. Finally we can get the fire detection system without using the heuristic fixed threshold values.

\subsection{Binary mask for background removal}

If we get the fire color range in a frame of input video sequences, there might be the fire-like color region in a scene. Therefore, first, it needs to remove the fire-like color region of background from thefire candidate regions. Figure 2 shows fire-like color region of the scene .

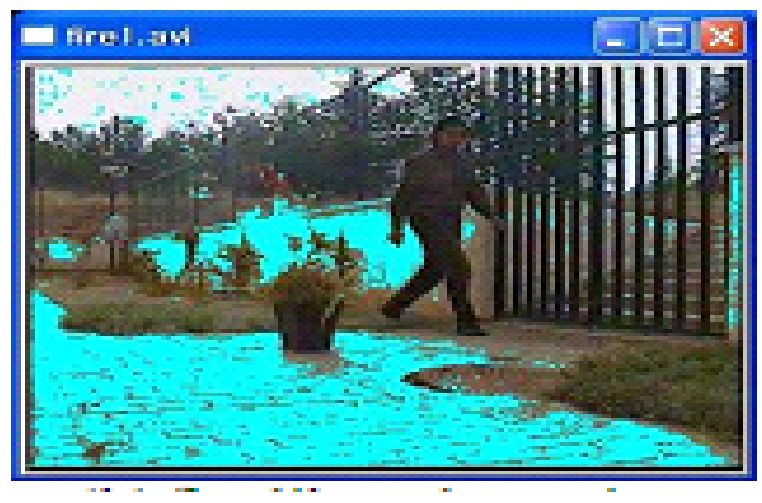


In the figure, the wall and board blocks have the fire-like color, so they are detected as the fire region. The image processing-based fire detection system can use the effect of background removal to avoid this kind of the fire-like color region.

Figure 3 depicts the binary background removal mask generation. In the figure, normal image means the normal scene without any fire. The first frame of video sequences before the fire occurrence may be used for that purpose.

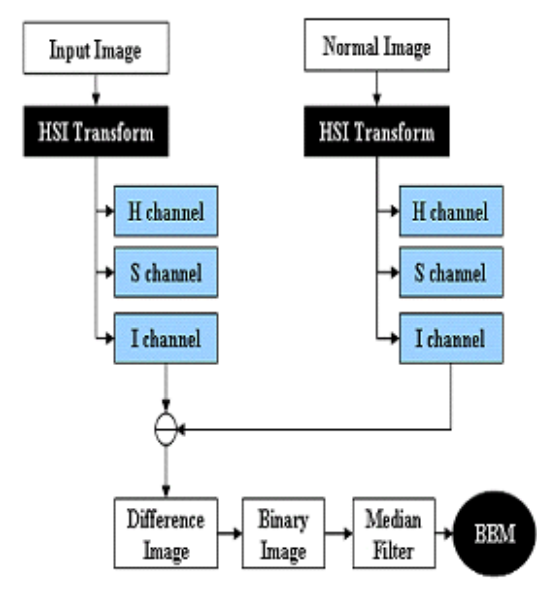

Figure 3. Binary mask generation for background

An input image and the normal image are first transformed into HSI. We get the difference image from I channel and make it binary form. There may be a lot of isolated spots, so we do median filtering to remove them. Finally we get the binary background mask (BBM) for the calculation of the statistical values of fire region.

\subsection{Automatic threshold with statistic values}

We use the following three rules to decide the fire pixel from the input image pixels. In the rules, av $\mathrm{R}$ is the average pixel value of the fire pixels through BBM. av $\mathrm{S}$ is the average value of saturation of the fire pixels. Therefore, all the heuristic fixed threshold values used in [3] are replaced with the statistical values which can be calculated automatically. Only the image pixels in the moving region among the input image pixels can be applied to the three rules one by one. And if an input pixel satisfies all the rules, it belongs to the fire pixel, otherwise, the non-fire pixel even if it is located in a moving region.

\section{Experiment and Results}

They developed the image processing-based fire detection system by using Visual $\mathrm{C}++6.0$ and OpenCV computer vision library. Windows XP, Core2Duo $1.86 \mathrm{GHz}$ and RAM $1 \mathrm{~GB}$ are used as our computing environment. The test video clips are FireClips from Signal and Image Processing Group at Bilkent University in Turkey (http://signal.ee.bilkent.edu.tr/ VisiFire/Demo/FireClips/). They noticed that it is not easy to precisely implement and compare the methods each other, because some of values of the methods are not clearly appeared in the papers.In figure,method 2 detects fire but it has many false positive regions.

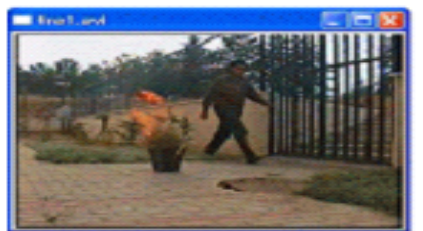

(a)

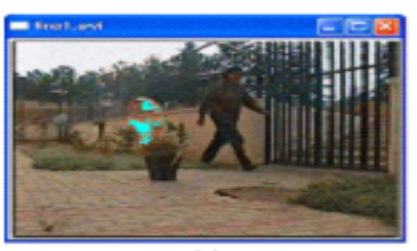

(c)

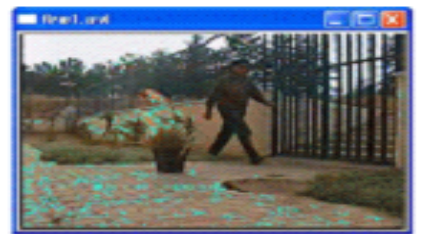

(b)

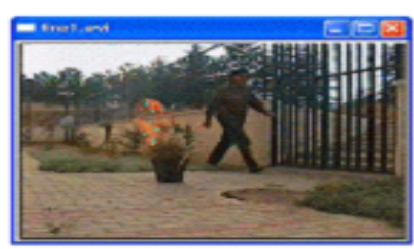

(d)

Figure 6. Example of video clip \#2: (a) method 1, (b) method 2, (c) method 3, (d) proposed 


\section{Conclusion}

This research is about the developing the automatic fire detection system using the statistic color model. Most of previous methods had some heuristic fixed threshold values, so if the input scenes are changed, it is not easy to detect fire in certain cases. They developed a fire detection method without the heuristic fixed threshold values. They modified Chen's methods using the statistical values that can be calculated automatically. As the experimental result using 600 frames from 6 video clips of sized 320x240 or 400x255, we could detect the fire region from the scenes without the fixed heuristic values. The experiment showed about average $85 \%$ detection rate without false positive in our proposed fire detection system. This method showed better detection rate, compared with other methods. It also had relatively lower detection rate in the video clips of small fire regions than that of video clips of large fire regions.

\section{References}

[1] Korean National Emergency Management Agency, Analysis Report of Fire Occurrence in January in $2007,2007$.

[2] Podrzaj and H. Hashimoto, "Intelligent Space as a Framework for Fire detection and Evaluation," Fire Technology, 2007.

[3] T.-H.(C.-H.) Chen, P.-H. Wu, and Y.-C. Chiou, "An Early Fire-Detection Method Based on Image Processing," International Conference on Image Processing, pp. 1707-1710, 2004.

[4] B.-M. Lee and Dongil Han, "Real-Time Fire Detection Using Camera Sequence Image in Tunnel Environment," Proceedings of ICIC, vol. 4681, pp. 1209-1220, 2007.

[5] T. Celik, H. Demirel, H. Ozkaramanli, and M. Uyguroglu, "Fire Detection using Statistical Color Model in Video Sequences," Journal of Visual Communication \& Image Representation, vol. 18, pp.176-185,2007.

[6] I. Kopilovic, B. Vagvolgyi, and T. Sziranyi, “Application of Panoramic Annular Lens for Motion Analysis Tasks: Surveillance and Smoke Detection," Proceedings of the International Conference on Pattern Recognition, 2000.

[7] B.U. Toreyin, Y. Deleoglu, U. Gudukbay, and A.E. Cetin, "Computer Vision Based Method for Real-time Fire Detection," Pattern Recognition Letter, vol. 27, pp. 49-58, 2006.

[8] Darko Stipanicev, Tomislav Vuko, Damir Krstinic, Maja Stula and Ljiljana Bodrozic, “Forest Fire Protection byAdvanced Video Detection System - Croatian Experiences,"Workshop on Improvement of Disaster Management Systems - local and global trends Trogir : RCADR Divulje ,2006. 